\title{
ATC/DDD Method to Assess the Injectable Antibiotics Utilization in a Tertiary Care Teaching General Hospital in India
}

\author{
Dr.Smita Anand Tiwari ${ }^{1}$, Dr.Ghongane Balasaheb Baburao ${ }^{2}$ \\ ${ }^{1}$ (Asstt.Prof.Department of Pharmacology, B.J.Government College, Pune,Maharashtra,India) \\ ${ }^{2}$ (Prof. \& Head of Department of Pharmacology, B.J.Government College, Pune,Maharashtra,India)
}

\begin{abstract}
Using the ATC/DDD index to evaluate the utilization of antibiotics is a standard method and helps in comparison across wards and hospitals. This study was conducted in a government hospital with 1190 beds (which excluded pediatrics ward).Data was recorded over twelve months from January 2013 to December 2013. Antibiotic use was evaluated using the ATC classification and DDD/100 bed-days formula and cost analysis was also done on 6 monthly basis along with restriction on antibiotic consumption (ie. January 2013June 2013 and July2013 -December 2013). Ceftriaxone sodium was the maximally used parentral antibiotic (2.607 and 2.135 DDD/100 bed-days) followed by Cefotaxime and Amikacin. Whereas Colistin and Linezolid were least used. Change in trends of utilization pattern was observed with certain antibiotics along with significant reduction in costs on antibiotics Rs.21,14,952 ( $p=0.011)$. Consumption of some injectable antibiotics did not vary much as they were integral part of treatment as per local sensitivity data and were used empirically. But the consumption of antibiotics used for hospital acquired infections needed to be monitored as they have a liability to be overused and monitoring may help in major cut down of budget consumption too.
\end{abstract}

Keywords: antibiotic consumption, ATC-DDD, DDD/100 bed-days, drug utilization, injectable antibiotic.

\section{Introduction}

The in-hospital use of various antibiotics is an ever growing concern for several reasons. Costly yet indispensible, antibiotic drugs attribute to a major proportion of the inflating drug budget. In hospitals this poses challenges for the administrators as well as purchase officers of health care services .[1] Main aim of any drug utilization research is to assess the rational use of drugs. To achieve this aim, drug audit at regular intervals may be inevitable. Drug utilization studies can be classified as descriptive and analytical studies. Descriptive studies assess and elaborate patterns of drug utilization with an aim to identify grey areas of research in this field. Analytical studies co-relate data on drug utilization to outcome of treatment, hospital morbidity, resistance patterns and quality of health-care, thus achieving the aim of rationalizing the therapy. Drug utilization research not only provides insight to the rationality of drug use but also hints whether a certain drug therapy provides value for money. The results of such pharmaco-economic studies, help to set priorities for the appropriate allocation of health care budgets.[2]It is therefore an important tool to study the use of drugs in clinical settings in various populations and its impact on health-care system as a whole.[3,4] The cost of health care is a matter of major concern in developing countries.[5] Antibiotic drug utilization patterns are necessary for a constructive approach to address issues that arise from availability of multiple antibiotics. [6] Studies have concluded that ,although in most cases the choice of antibiotics is in accordance with the guidelines which is especially true for leading medical centers; yet there was still unwarranted overuse of prophylactic agents which may account to increased costs to the hospital. [7] The problem may turn graver with the development of resistance to antibiotics, ecological imbalances and super-infections in various patients. [8,9] Main limitation in drug utilization study is that each formulation has different unit dose of daily administration, therefore a specific standardized method should be used in the evaluation of in-hospital antibiotic use. Nordic Council on Medicines (Uppsala, Sweden) along with Norwegian researchers developed the DDD is a measurement unit that was in 1976. The DDD does not necessarily mean recommended or prescribed daily dose for any patients but is simply the assumed average maintenance dose per day for a drug in its main indication and route, for adults. It is a measurement unit recommended to be used during drug utilization studies. [10]

The ATC (anatomic-therapeutic-chemical) classification is a classification that assigns code letters and numbers to drugs as per their pharmacological category. DDD is be assigned only for drugs that already have an ATC code. DDD although may not reflect the prescribed daily dose as mentioned previously, however they provide a standard unit of measurement independent formulation, units, dosing schedule . They hereby enable the researcher to compare between variety of population groups. DDD/100 bed-days is an estimate of consumption of drugs among hospitalized patients. [11,12] The present study therefore evaluates the injectable antibiotic utilization as six-monthly trends in a Government set-up of a tertiary care teaching hospital in India. 
The aims of the study were:

(a) To assess the use of parenteral/injectable antibiotics, in a tertiary care government hospital in India on sixmonthly basis over one year.

(b) To assess the impact of such variation in antibiotic utilization on expenditure over antibiotics by the drug store.

\section{Material And Methods}

This was a partly prospective and partly retrospective drug utilization study. It was conducted in a, 1190 bedded government tertiary care teaching hospital (excluding pediatric wards). The study is a part of a larger study that was approved by the ethics committee (ethical app no: BJMC/IEC/Pharmac/1210115-17, dt:2312-2010).And was done in six monthly phases as January 2013 to June 2013 and July 2013 to December 2013 .The data for this study was over twelve months and missing and cost data was recorded retrospectively. Data on drug utilization in terms of dose, duration and indications was recorded from antibiotic indent forms, patient records and drug store records. Only total amount of antibiotic consumed patient-wise and ward-wise was noted. Data missing due to technical limitations was collected retrospectively.

The drug utilization was computed as DDD/100 bed-days.[13,14,15] The data was evaluated by Microsoft Excel software for analysis.

DDD/100 bed-days $=$ Drug consumption in the study period $(\mathrm{mg}) \times 100$

$$
\text { Assigned DDD (mg) } x \text { ( Period of study } \times \text { Bed strength } \times \text { Average occupancy) }
$$

The expenditure on antibiotics was calculated according to the rate Government rate-contract prices of antibiotics available with the drug store. These prices remains constant for three years.

Cost Difference $=\mathrm{A}-\mathrm{B}$

Where- A (Jun.2013to Dec.2013) $=\Sigma($ total vials consumed of injectable antibiotic per antibiotic under study $\mathrm{x}$ price per unit/vial)

B (Jan.2013to Jul.2013) $=\Sigma$ (total vials consumed of injectable antibiotic per antibiotic under study $\mathrm{x}$ price per unit/vial) [16]

\section{Results}

A total of 64,786 in-patients were recorded in the year 2013 .[Fig.1] Total 30973 patients from January 2013 to June 2013 and 33813 in-patients in the period of July 2013 to December 2013. Treatment period ranged from 1-16 days for patients and was rounded off to an average of six days in all the study months. These parameters were used for calculating bed occupancy.

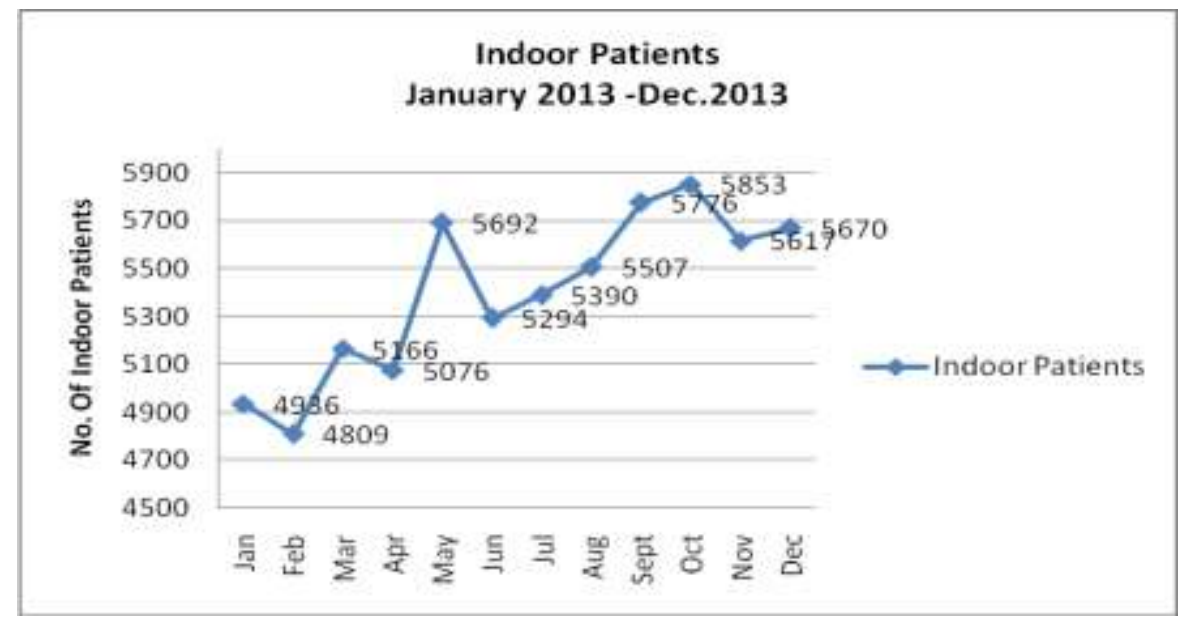

Figure 1: Indoor Patients in the year month-wise

Ceftriaxone sodium was the most utilized injectable antibiotic amounting to $2.607 \mathrm{DDD} / 100$ bed-days in Jan.2013-Jun.2013 and 2.136 DDD/100 bed-days in Jul.2013-Dec.2013. The second most commonly used antibiotic in the period Jan.2013-Jun.2013 was Cefotaxime (1.761 DDD/100bed-days) whereas Amikacin (1.438 DDD/100 bed-days) was the second most commonly used antibiotic in the period Jul.2013-Dec.2013.Colistin was the least used injectable in both the periods (0.000 and 0.00024 DDD/100 bed-days ) followed Linezolid. (Table-1) There was a definite decrease in the utilization of injectables like - Imipenem + Cilastin , Clarithromycin, Linezolid, Meropenem, Piperacillin + Tazobactum, Teicoplanin and Vancomycin. (Table1 ,Fig.2) 
ATC/DDD method to assess the injectable antibiotics utilization in a tertiary care teaching general ..

Table-1 -Injectable antibiotic consumption in the year 2013 as six monthly assessment:

\begin{tabular}{|c|c|c|c|c|}
\hline Injectable Antibiotic & ATC Code & $\begin{array}{l}\text { Assigned } \\
\text { DDD (D) }\end{array}$ & $\begin{array}{l}\text { Jan2013- } \\
\text { Jun2013 }\end{array}$ & $\begin{array}{l}\text { July2013- } \\
\text { Dec2013 }\end{array}$ \\
\hline Amoxycillin+Clavulanic acid & J01CR02 & 3 & 0.198 & 0.189 \\
\hline Imipenem + Cilastatin & J01DH51 & 2 & 0.045 & $0.012^{@}$ \\
\hline Clarithromycin $500 \mathrm{mg}$ & JO1FA09 & 1 & 0.079 & $0.032^{@}$ \\
\hline Colistin 10 lac units (DDD-3mu) & J1XB01 & 3 & $0.00000^{\$}$ & $0.00024^{\$}$ \\
\hline Linezolid $600 \mathrm{mg}$ & J1XX08 & 1.2 & 0.010 & $0.006^{@}$ \\
\hline Meropenem $500 \mathrm{mg}$ & J01DH02 & 2 & 0.012 & $0.009^{@}$ \\
\hline Meropenem $1000 \mathrm{mg}$ & J01DH02 & 2 & 0.039 & $0.014^{@}$ \\
\hline Piperacillin + Tazobactum & J01CR05 & 14 & 0.254 & $0.184^{@}$ \\
\hline Teicoplanin & J1XA02 & 0.4 & 0.017 & $0.003^{@}$ \\
\hline Vancomycin & J1XA01 & 2 & 0.084 & $0.077^{@}$ \\
\hline Amikacin & J01GB06 & 1 & $1.442 * * *$ & $1.438 * *$ \\
\hline Cefoperazone+ Sulbactum & J01DD12 & 4 & 0.006 & 0.004 \\
\hline Cefotaxime & J01DD01 & 4 & $1.761 * *$ & $1.149 * * *$ \\
\hline Ceftazidime & J01DD02 & 4 & 0.051 & 0.062 \\
\hline Ceftriaxone sodium & J01DD04 & 2 & $2.607 *$ & $2.136^{*}$ \\
\hline
\end{tabular}

*Most commonly utilized injectable antibiotic, ${ }^{* *}$ second most used and $* * *$ third most commonly used.\$ least used injectable antibiotics. ${ }^{\circledR}$ Drastic decrease in utilization.

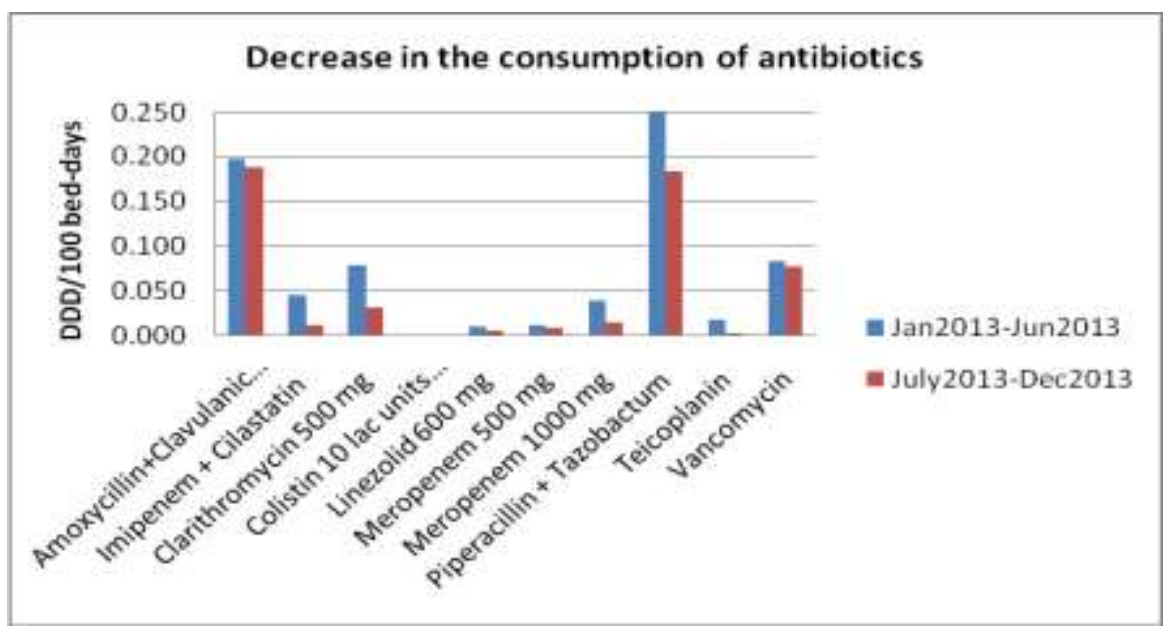

Figure 2-Decrease in consumption of selective antibiotics in the year 2013

The expenditure done on injectable antibiotics from Jan.2013 -Jun.2013 was Rs.8061901.14 (INR).As the consumption of antibiotics decreased in the next six months (i.e. Jul.2013 - Dec. 2013), the expenditure done on these drugs also decreased to Rs.5646954.65 (INR). (Table 2, Fig. 3)A total reduction in cost by Rs.21,14,952 on the same antibiotics.

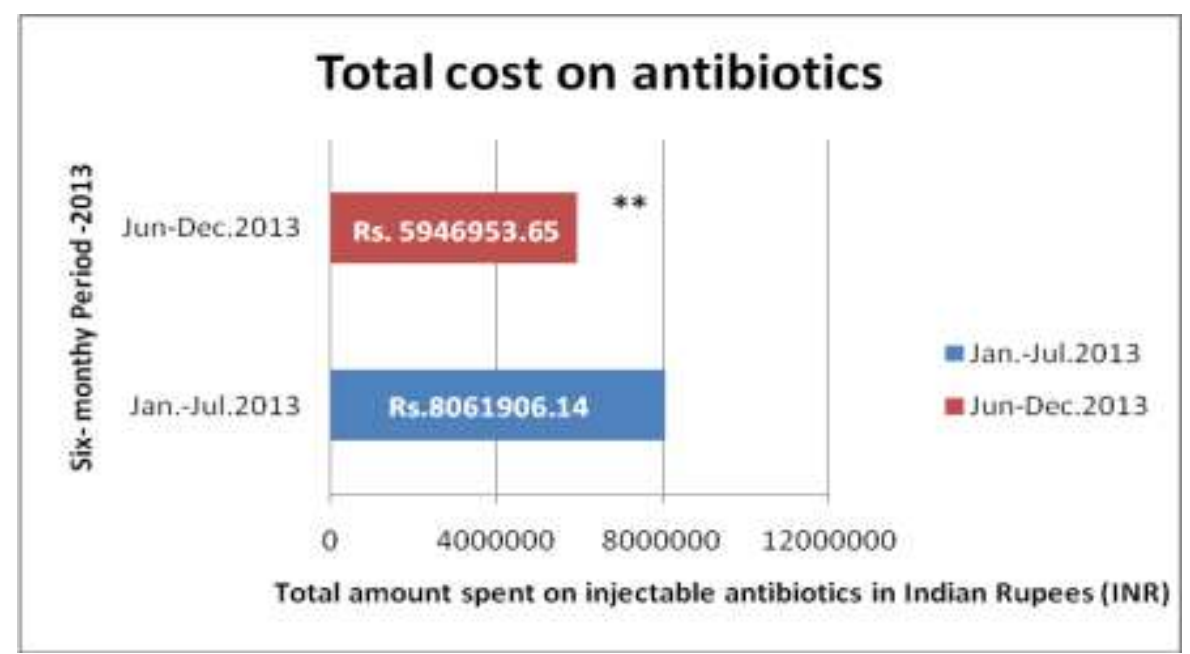

$* *$ p-value $=0.011$.

Figure-3: Difference in the total amount spent on antibiotics in the year 2013 -a six monthly assessment 
Table-2: Drug-wise cost of antibiotic in the year 2013 -as six monthy analysis

\begin{tabular}{|l|r|r|}
\hline \multirow{2}{*}{ Name of Antibiotic } & \multicolumn{2}{|c|}{ Total Expense in Rupees } \\
\cline { 2 - 3 } & Jan to Jun-2013 & Jul to Dec-2013 \\
\hline Amoxycillin+Clavulanic acid & 248265 & 258525 \\
\hline Imipenem + Cilastatin & 598636.35 & 170826.75 \\
\hline Clarithromycin 500 mg & 692384 & 305671.8 \\
\hline Colistin 10 lac units & 0 & 49170 \\
\hline Linezolid 600 mg & 15480 & 10656 \\
\hline Meropenem 500 mg & 103472.25 & 90858.49 \\
\hline Meropenem 1000 mg & 231006.62 & 92561.78 \\
\hline Piperacillin + Tazobactum & 2147484.4 & 1701513.4 \\
\hline Teicoplanin & 263580 & 47610 \\
\hline Vancomycin & 158775.52 & 159158.83 \\
\hline Amikacin & 289440 & 315000 \\
\hline Cefoperazone+ Sulbactum & 4544 & 3200 \\
\hline Cefotaxime & 1350888 & 961961.6 \\
\hline Ceftazidime & 68400 & 1690000 \\
\hline Ceftriaxone sodium & 1889550 & 5946954 \\
\hline Total Cost on anitbiotics & 8061906 & 2114952 \\
\hline Cost Difference & \multicolumn{2}{|c|}{} \\
\hline
\end{tabular}

\section{Discussion}

Antibiotic consumption of any hospital is a dynamic data that is governed by many factors like change in sensitivity patterns of various local microbes, addition of new drug molecules in the market, their availability in hospital pharmacy/drug stores and changing treatment guidelines. Therefore antibiotic consumption studies are done mainly to identify areas of improvement, to compare antimicrobial use across wards and hospitals, [17] to study impact of interventions [16] and capture attention of healthcare professionals on its rational use. [18]

The data of the present study was recorded over twelve months .In the first six months i.e January to June, a total of 30973 in-patients were recorded; whereas there was an increase in number in in-patients in the next six months (33813 in-patients). The antibiotics in both the periods antibiotics were most frequently used for post -surgical patients $(29.25 \%$ and $21.16 \%$ respectively) by various surgical departments (viz. surgery, orthopedics and obstetrics - gynecology). Deshmukh V et al., also report maximum antimicrobial utilization done for surgical chemoprophylaxis [19]. Ceftriaxone sodium was the most utilized injectable antibiotic amounting to 2.607 DDD/100 bed-days in January-June 2013 and 2.136 DDD/100 bed-days in July-December 2013. This finding is in accordance with the results of another study ,but the researchers in that study included Ceftriaxone and combination of Ceftriaxone + Sulbactum collectively for reporting .[20] A study by Mittal et al concluded that beta-lactams were maximally used ,but the researchers had included cephalosporins collectively in beta-lactam group. [21] A study by Paul et al also concludes the high use of Ceftriaxone . [22] Amikacin (1.438 DDD/100 bed-days) was the second most commonly used antibiotic in the period July -December 2013 as most of the utilization was by surgical patients.A high use of Amikacin was reported by Tripathi et al. in laprotomy patients . [23] Colistin was the least used injectable in July- December. period ( 0.00024 DDD/100 bed-days ) followed Linezolid as both are reserve antibiotics for resistant pathogens. Colistin use could not be commented in the January.-June 2013 period as it was not available in the drug store in that period. There was a definite decrease in the utilization of injectables like - Imipenem + Cilastin , Clarithromycin, Linezolid , Meropenem, Piperacillin + Tazobactum, Teicoplanin and Vancomycin. During the study period several restrictions were imposed on the indent of these drugs, therefore issued only if use was justified and rational as per the culture sensitivity. This reflected as a gradual but a definite decrease in utilization of these drugs. Antibiotic restrictions although are not favored by certain authors [24,25] ; yet if the restrictions are imposed for rational utilization of antibiotics it is still justified. There was a significant reduction in costs on antibiotics Rs.21,14,952 ( $\mathrm{p}=0.011$ ) in the period June to December although the inpatient admissions had increased. This study is a part of a larger study which takes into consideration the change in resistance patterns and patient outcome patterns due to antibiotic restrictions.

\section{Conclusion}

The present study concludes that Ceftrixone was the most commonly used injectable antibiotic followed by Amikacin in the year 2013 in our hospital. Consumption of these injectable antibiotics did not vary much as they are integral part of treatment as per local sensitivity data and are used empirically. The reserve drug like Colistin and Linezolid are used sparingly as per their indications. Restriction on antibiotics take time to show effect on utilization patterns hence a six-monthly analysis should be done to overview changes but definitely helps in resource limited setting and may help to minimize cost while concurrently assisting in providing optimum healthcare. The limitation of the study was that it could not comment on the change in the resistance patterns due to formulary restrictions. 


\section{References}

[1]. Kunin CM, Staehr Johansen K, Worning AM, Daschner F.Report of a symposium on use and abuse of antibiotics worldwide. Rev Infect Dis 1990; 12: 12-9.

[2]. Introduction to drug utilization research / WHO International Working Group for Drug Statistics Ibnosina Journal of Medicine and Biomedical Sciences (2012) Ibnosina J Med BS 19Methodology, WHO Collaborating Centre for Drug Statistics Methodology, WHO Collaborating Centre for Drug Utilization Research and Clinical Pharmacological Services. Geneva: World Health Organization; 2003. p. 8-12.

[3]. Shankar PR, Upadhyay DK, Subish P, Bhandari RB, Das B. Drug utilisation among older inpatients in a teaching hospital in Western Nepal. Singapore Med J. 2010;51:28-34

[4]. Sachdeva PD, Patel BG. Drug utilization studies - Scope and future perspectives. Int J Pharm Biol Res. 2010;1:11-7.)

[5]. Kuruvilla A, George K, Rajaratnam A and John KR: Prescription patterns and cost analysis of drugs in a base hospital in South India .Natl Med J India 1994, 7:167-168)

[6]. Srishyla MV, Naga Rani MA and Venkataraman BV: Drug utilization of antimicrobials in the in-patient setting of a tertiary hospital .Indian J Pharmacol 1994, 26:282-287.

[7]. Gordana Pešić, Zorica Jović, Karin Vasić Application Of The Atc/Ddd Methodology To Compare Antibiotic Utilization In Two University Hospital Surgical Departments. Medicine and Biology .Vol.12, No 3, 2005, pp. 174 - 178.

[8]. Cizman M: The use and resistance to antibiotics in the community. Int J Antimicrob Agents 2003, 21:297-307.

[9]. Kunin CM, Johansen KS, Worming AM, Daschner FD: Report of a symposium on use and abuse of antibiotics worldwide. Rev Infect Dis1990, 12:12-16.

[10]. Dominique L. Monnet .Measuring Antimicrobial Use: The Way Forward, Clinical Infectious Diseases 2007; 44:671-3, 2007 by the Infectious Diseases Society of America. Downloaded from http://cid.oxfordjournals.org/ by guest on May 3, 2013

[11]. WHO Collaborating Centre for Drug Statistics Methodology:Guidelines for ATC classification and DDD assignment Oslo 2002.

[12]. Ravi Pathiyil Shankar, Praveen Partha , Nagesh Kumar Shenoy,Joshy Maducolil Easow and Kottallur Narayanan Brahmadathan.Prescribing patterns of antibiotics and sensitivity patterns of common microorganisms in the Internal Medicine ward of a teaching hospital in Western Nepal: a prospective studyAnnals of Clinical Microbiology and Antimicrobials 2003, 2:7 (This article is available from: http://www.ann-clinmicrob.com/content/2/1/7)

[13]. Monnet DL, ABC Calc - antibiotic consumption calculator [Microsoft Excel application], version 3.1 Copenhagen, Denmark: Statens Serum Institut; 2006. Available at: http://www.escmid.org/esgap.

[14]. Oslo: Norwegian Institute of Public Health. WHO Collaborating Centre for Drug [5] Statistics Methodology. Structure and principles [Internet]. Vol. 2006. Available from: http://www.whocc.no/atc/structure_and_principles/

[15]. Gyawali S, Shankar PR, Saha A, Mohan L. Study of prescription of injectable drugs and intravenous fluids to inpatients in a teaching hospital in Western Nepal. McGill J Med. 2009;12(1):13-20.

[16]. Smita Anand Tiwari, Balasheb Baburao Ghongane, Bharti Ramchandra Daswani, Sangeeta Sanjay Dabhade. Restricted Parenteral Antibiotics Usage Policy in a Tertiary Care Teaching Hospital in India.Journal of Clinical and Diagnostic Research. 2017 May, Vol-11(5): FC06-FC09, DOI: 10.7860/JCDR/2017/24048.9776.

[17]. James M Hutchinson, David M Patrick, Fawziah Marra, Helen Ng,William R Bowie, Laurie Heule et al. Measurement of antibiotic consumption:A practical guide to the use of the Anatomical Therapeutic Chemical classification and Defined Daily Dose system methodology in Canad,.Can J Infect Dis Vol 15 No 1 January/February 2004,29-35.

[18]. Sözen et al.: Application of ATC/DDD methodology to eveluate of antibiotic use in a general hospital in Turkey. Annals of Clinical Microbiology and Antimicrobials 2013 12:23.

[19]. Deshmukh V, Khadke V, Patil A, Lohar P. Study of prescribing pattern of antimicrobial agents in indoor patients of a tertiary care hospital. Int J Basic Clin Pharmacol [Internet]. 2013;2(3):281-85.

[20]. Aanchal Wats, Sumit SohalTrends of Antibiotic Use Among the Indoor Patients of Medicine and Pediatric Ward at A Tertiary Care Hospital .International Journal of Science and Research (IJSR) www.ijsr.net, Volume 4 Issue 9, September 2015,229-232.

[21]. Niti Mittal, R. Mittal, I. Singh, Nusrat Shafiq And S. Malhotra. Drug Utilisation Study in a Tertiary Care Center: Recommendations for Improving Hospital Drug Dispensing Policies. Indian J Pharm Sci 2014;76(4):308-314.

[22]. Suhrita Paul, Tithishri Kundu, Pragnadyuti Mandal, Manab Nandy and Nidhi Agrahari ,Antibiotic Utilisation Pattern In Indoor Patients Of Medicine Department In A Tertiary Care Centre In Eastern India. World Journal of Pharmaceutical Research ,Volume 5, Issue 7, 1902-1909.

[23]. Hiren M. Chawda, Mahendra Patel, Divyesh Mandaviya, Manish Barvaliya, Tejas Patel, Dr. C. B. Tripathi. Utilization Pattern Of Antimicrobials Among Patients Undergone Midline Laparotomy In Four Tertiary Care Teaching Hospitals Of Gujarat, India: Multicentric Retrospective Study. Asian J Pharm Clin Res, Vol 6 Suppl 5, 2013, 145-150.

[24]. Garau J,Impact of antibiotic restrictions: the ethical perspective. Clin Microbiol Infect. 2006 Aug;12 Suppl 5:16-24.

[25]. Power E, Impact of antibiotic restrictions: the pharmaceutical perspective. Clin Microbiol Infect. 2006 Aug;12 Suppl 5:25-34. 